\title{
Performance Comparison of QEC Network based JAVA Application and Web based PHP Application
}

\author{
Sanaullah Memon ${ }^{1}$ \\ Software Engineering Department \\ Mehran UET, Jamshoro, Sindh, Pakistan \\ Rasool Bux Palh ${ }^{2}$ \\ SibiSoft Technologies \\ Karachi, Sindh, Pakistan
}

\author{
Muniba Memon ${ }^{3}$ \\ Computing Department \\ Indus University Karachi, Sindh, Pakistan \\ Hina Siddique Memon ${ }^{4}$ \\ Department of Computer Science \\ Shah Abdul Latif University Khairpur, Pakistan
}

\begin{abstract}
Every organization wants to automate the manual system for moving and storing their data in particular format. A QEC department takes feedback of teacher evaluation manually from the students in the university that is somehow more difficult to maintain the record of a teacher, more cost-effective and fewer chances to generate an accurate and optimized report. The computerized system has been developed that generates an accurate and optimized report, easy to maintain the record of the teacher. Lots of possibilities are available to design and develop the application using different programming languages. We have developed a network-based JAVA application and web-based PHP application to automate the manual system of teacher evaluation. The GUI of the application contains 18 questions as per policy of HEC which will be answered by the students. After submitting the answers to questions to the server, an excel report will be ready to generate. Our primary focus is to measure the performance of the server of a network-based JAVA application and web-based PHP application. Both forms contain the same scenario, but here we have to find which form is more suitable and beneficent for an organization in terms of their server's performance parameters like average response time, throughput, and standard deviation and data transfer rate.
\end{abstract}

Keywords-QEC; network based JAVA; web based PHP; server; apache JMeter

\section{INTRODUCTION}

As the growing of software technology day by day, the software applications are needed for every organization to run their system correctly. Data is the bits of information, crossregion and comprehensive on the evaluation of environmental impact [1]. Framed collection of data is called database. Database management system facilitates the user to collaborate with databases for retrieving, managing and accessing the data. The database supply effective methods to stock the facts. There is research required to collar database system and to search approaches for system's knowledge-based technology [2]. By using the web interface and availability of resources and rapid growth of internet, a user can easily access the database by performing operations on it. The web has a medium containing huge data where the user can access it through the web [3]. The Internet has become an important key resource of an organization. The usage of internet service is now a matter of learning and analyzed as a dynamic process [4].

\section{BACKGROUND}

Data and Information are the synonym term to each other, but each contains accurate and exact meaning. There are two concepts to collect and manage the data in the database either manually or systematically. To receive and calculate the data manually and to maintain and generate the optimized report is more quite tricky.

In order to create and manage data of the QEC in an organization, there is a need for the computerized system. The automated system is an excellent solution to manage information. The aim to develop a QEC application is to automate the manual system of teacher evaluation. Many resources are required to get teachers evaluation, and lots of analysis is necessary to generate the excel report, and there were thousands of papers were distributed among students to get their teachers evaluation which was somehow more difficult to arrange a number of sets needed for assessment of the students, but this computerized system replaces the manual systems.

The two most popular programming languages JAVA and PHP contain the open source for designing and developing different applications. Comparing both languages, this paper analyzes that the interoperability support of JAVA is greater than PHP. JAVA takes more time to program, but it is a stable application, covers much security problems and gives better influences [5]. Strongly typed language such as JAVA expressed their ability to produce robust, easily maintainable applications while lightweight language such as PHP is critical to provide infrastructure for component-based applications [6].

The GUI (Graphical User Interface) of the application contains only 18 question as per policy of HEC which will be answered by the student. After submitting these answers to the server, an excel report will be ready to generate.

\section{LIFE CyClE OF QUALITY ENHANCEMENT CELL APPLICATION}

To describe the possible environmental effect of the system, a life cycle assessment is performed [7]. The application mainly consists of three parts described below: 
- Server Program: The server program is a server-side program which machine must be running. Whenever any of batches is to be evaluated for assessment of teacher and on same machine database file exists, all results submitted by the student from the client will be stored in database file using server program.

- Client Program: The client application is part of QEC application which will be running on LAN (local area network), i.e. (Computer Lab) to evaluate teacher using QEC application.

- Admin Panel: Admin panel will reside on the server side to manage the admin related task like inserting teacher, deleting teacher from the database and setting up that for which batch we are getting the evaluation from the students. Due to the significant distance between a client-side application and server-side application factor, the quality of service of networkbased application may demean [8].

\section{FEATURES OF APPLICATION}

- Accuracy: The application generates excel report which is $100 \%$ accurate as per the result submitted by the students the manual report may not be accurate because that is calculated by any human and human can make an error,but there is no any chance for error in the computer-generated report.

- Financial Benefit: In manual to get an assessment of any teacher, there is need of a lot of papers which were to be distributed among the students, and they provide their answer on paper after getting these all paper these were submitted to QEC, and then QEC use to start the process to generate a report.

- Time-saving in terms of Generate Assessment Report: The report is exported to excel file which exposes the result of all teachers of one department this report is generated within 3 seconds whereas in the manual system it may take about a number of days for a single department to generate the same report.

- Dynamic Report: The program written for generating the report is smart enough to createdynamic report. Dynamic means whatever result is submitted by the students the program will read a database file and prepares the report accordingly and exports that report to excel. The report is totally depending on the result being submitted by the students.

- User-friendly Application: Application is straightforward to use because it does not require any input from students except the answer of question all other information is maintained by application itself on runtime suppose student from any department has finished his evaluation for one teacher then he need not select another teacher only he presses Submit \& Proceed button then application submits these answers to server and selects another teacher for the same students and in last when the students completed evaluation for all teachers Submit \& Proceed button goes disable, and message is being displayed the students "Your Feedback submitted successful Thanks for your usual co-operation" after this that students feedback entirely submitted and save to server machine.

- Maintain log file at server side: Any computer connected to server program is being registered with its computer name in log file name ComputerList.log also it displays the state of the client whether the connected client has completed the teacher evaluation or still working on Performa suppose the connected client name is John then its entry in the log file will be like 01 . John NO here John is the name of connected client NO means this client is still working on Performa as soon as this client completes the Performa the status of that client is changed from NO to YES. YES means this client has completed filling Performa.

- Application is configurable: Service-oriented architecture is developed for configurable application packages for the use [9]. Most of the parameters are configurable in demand; admin does not need to change the code at any point a file named config.properties provide the feasibility to change any parameterby changing the code base.

- Robustness of application: The change effects are related to the robustness which is before and after a change has occurred, scaled differences in system parameters [10]. The robustness of application maintains the client state on the server side.

A leading web based language development language PHP organizes without particular course of action about things or structure [11]. Attachment modifying is used to correspond those you quit offering on that one PC to an alternate machine with one another clinched alongside An p2p framework [12]. The java system built modifying will be very basic Furthermore not difficult Yet remains puzzling [13].

There are two phases in QEC application as shown in Table I.

- Server Application Files

- Client Application Files

These two phases are strictly worked with each other.

Database details used in the application are shown in Table II. 
TABLE II. QEC APPLICATION TeChNICAL DETAILS

\begin{tabular}{|c|c|c|c|c|}
\hline \multicolumn{5}{|c|}{ SERVER APPLICATION FILES } \\
\hline S\# & FILE NAME & FILE TYPE & LOC & PURPOSE \\
\hline 1. & QEC Server & JAVA & 316 & $\begin{array}{l}\text { Start server and handles all requests } \\
\text { coming from multiple clients. }\end{array}$ \\
\hline 2. & Generate Teacher Evaluation Report & JAVA & 508 & $\begin{array}{l}\text { Generate dynamic report and } \\
\text { export to excel }\end{array}$ \\
\hline 3. & Answers DTO & JAVA & 87 & Populate answers from database \\
\hline 4. & Departments DTO & JAVA & 33 & \\
\hline 5. & Teachers DTO & JAVA & 44 & Populate departments for database \\
\hline 6. & Application Utility & JAVA & 40 & $\begin{array}{l}\text { Its utility class which reads the } \\
\text { parameters from config.properties } \\
\text { and provide these parameters to } \\
\text { server program }\end{array}$ \\
\hline 7. & Database Manager & JAVA & 303 & Manages all database transaction \\
\hline 8. & Config.properties & Properties & 2 & $\begin{array}{l}\text { Maintain configurable parameters } \\
\text { of application }\end{array}$ \\
\hline 9. & Computer List & LOG & N/A & $\begin{array}{l}\text { Logs the computer name of client } \\
\text { connected to server }\end{array}$ \\
\hline \multicolumn{5}{|c|}{ CLIENT APPLICATION FILES } \\
\hline 1. & Main & JAVA & 23 & Starts Client application \\
\hline 2. & Teacher Evaluation Form & JAVA & 2909 & $\begin{array}{l}\text { User interface of client program } \\
\text { from where student fill the teacher } \\
\text { evaluation proforma- } 10\end{array}$ \\
\hline 3 & Today & JAVA & 109 & $\begin{array}{l}\text { Returns data time day and year } \\
\text { information }\end{array}$ \\
\hline
\end{tabular}

\section{FEATURE COMPARISON OF QEC APPLICATION}

To compare the features of QEC application with the application which is already used in Shaheed Benazir Bhutto University Nawabshah, we have accepted the path of creating a list of required features.

Technical requirements of applications are shown in Table III, analyzes the applications on the basis of particular features and their availability, each feature can contain one of the following values that are: $\checkmark$ (yes, present), $\times$ (no, not present), + (present but limited use).

TABLE III. DATABASE DETAILS

\begin{tabular}{|l|l|l|l|}
\hline S\# & TABLE NAME & No: of Rows & $\begin{array}{l}\text { No: of } \\
\text { Columns }\end{array}$ \\
\hline 1. & Department & 3 & 2 \\
\hline 2. & Teachers & 9 & 5 \\
\hline 3. & Answers & 288 & 7 \\
\hline 4. & $\begin{array}{l}\text { MAINTAIN-EACH- } \\
\text { CLIENT-STATE }\end{array}$ & 23 & 4 \\
\hline
\end{tabular}

\section{AdVAntages OF DESKTOP AND WEB-BASED APPLICATIONS}

The advantages of desktop based and web-based application are shown in Table IV.

TABLE IV. FEATURE COMPARISON OF QEC APPLICATION

\begin{tabular}{|l|l|l|l|}
\hline CATEGORIES & FEATURES & QEC APP & $\begin{array}{l}\text { QEC APP } \\
\text { OF SBBU }\end{array}$ \\
\hline Interface & Login history & $\checkmark$ & $x$ \\
\hline Performance & Page Load & $\checkmark$ & $\checkmark$ \\
\hline Dynamic Report & Excel report & $\checkmark$ & $x$ \\
\hline Accuracy & Error Calculation & $x$ & + \\
\hline Development & Deployment & $\checkmark$ & + \\
\hline Maintenance & Log File & $\checkmark$ & $x$ \\
\hline
\end{tabular}

TABLE V. AdVANTAGES OF DESKTOP AND WEB-BASED APPLICATION

\begin{tabular}{|l|l|l|}
\hline S.No & $\begin{array}{l}\text { DESKTOP BASED } \\
\text { APPLICATION }\end{array}$ & $\begin{array}{l}\text { WEB BASED } \\
\text { APPLICATION }\end{array}$ \\
\hline$(1)$ & No reliance on an internet & Cross Platform \\
\hline$(2)$ & Much easier to customize & Huge Community \\
\hline$(3)$ & High Efficiency & Fast Development Cycle \\
\hline$(4)$ & User Interface Flexibility & Standard Based \\
\hline
\end{tabular}


TABLE VI. DISADVANTAGES OF DESKTOP AND WEB-BASED APPLICATION

\begin{tabular}{|c|l|l|}
\hline S.No & $\begin{array}{l}\text { DESKTOP BASED } \\
\text { APPLICATION }\end{array}$ & $\begin{array}{l}\text { WEB BASED } \\
\text { APPLICATION }\end{array}$ \\
\hline$(1)$ & $\begin{array}{l}\text { Speed at which Software } \\
\text { upgrades }\end{array}$ & Larger Overhead \\
\hline$(2)$ & $\begin{array}{l}\text { Restricted to a single standalone } \\
\text { machine }\end{array}$ & $\begin{array}{l}\text { Less control over computer } \\
\text { resources }\end{array}$ \\
\hline$(3)$ & Less Connectivity & Accessible any where \\
\hline
\end{tabular}

The disadvantages of desktop based and web-based application are shown in Table V.

\section{DATABASE SCHEMA}

In the framework of the traditional information system, database developing is at the center of an information system expresses a hard alimony problem [14]. Database Schema expresses the logical view of the whole database that describe show to manage and organize the data or information, and the relationship between them. It also explains all the set of rules that are to be enforced on data. A database schema defines its entities, attributes and the relationship between them. It contains descriptive details of the database that can be illustrated by the schema. A database designer designs a database schema that helps the developer or programmer to manage and understand the database and to make a useful database.

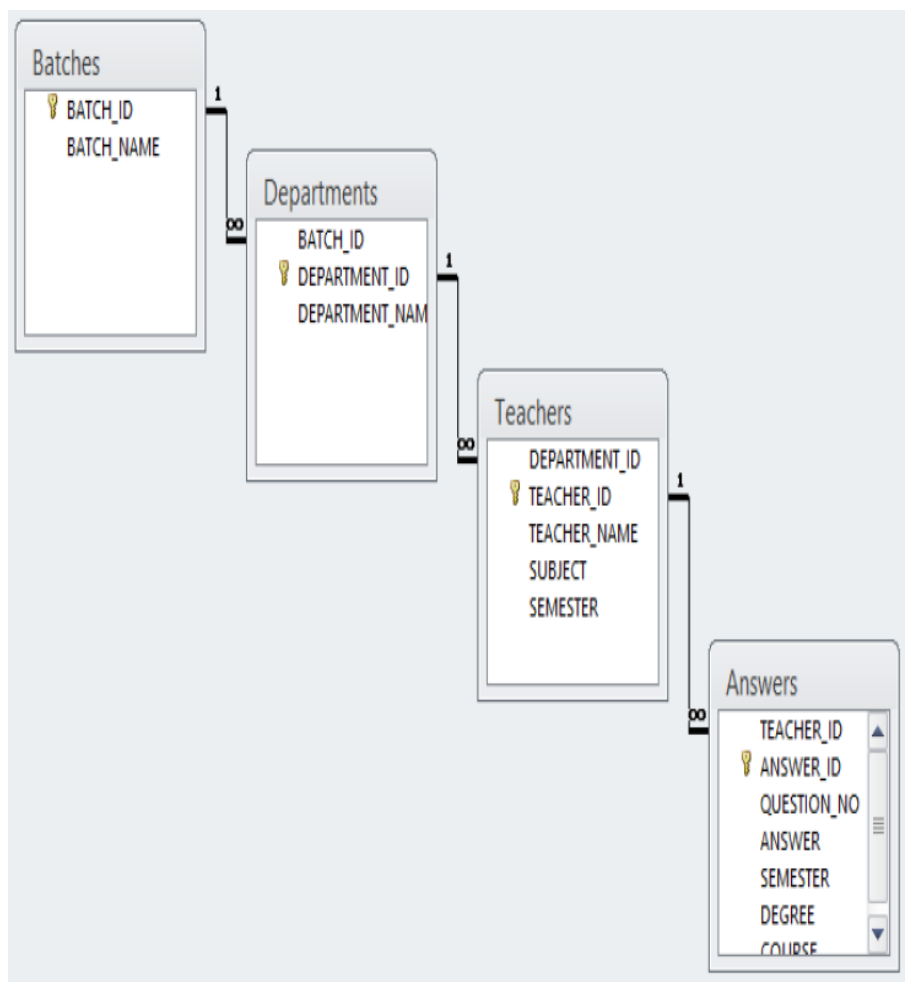

Fig. 1. Database Schema of the Application.
The databases used in QEC application have four tables, i.e., batches, departments, teachers and answers. These four tables have one to many relations with each other as shown in Fig. 1.

\section{QEC PROJECT DESIGN}

Designing a project plays an important role which follows the methods and theories that contest the behavior of end users and project goals [15]. The project is designed and developed to provide the advanced functionalities of feedback of teacher evaluation, which contains some teachers related questions as per policy by HEC and answered by the students during the assessment of feedback of teacher evaluation. This feedback evaluation system generates an accurate and optimized report in excel and provides correct results.

\section{QEC APPLICATION MAIN SCREEN}

This is the main view of QEC application that shows text boxes, check boxes, buttons and different contents of the application as shown in Fig. 2.

The text boxes contain specific information of the department's related Instructors and course titles which is handled by the system administrator. These boxes are already selected by the administrator when taking feedback of teacher evaluation from the students of the different batches of the departments. There is no any facility to make any changes in the text boxes to the student. The checkboxes show different options for each question. Each question contains some teacher's related meaning. The Graphical User Interface of this application provides only 18 items as per policy of HEC related with the teacher which will be answered by the student. By attempting all the questions of the teacher evaluation form, press Submit and proceed. The instructor name and course title have been changed dynamically after attempting and submitting all the questions related to the teacher and his course title. There is validation in attempting the entire questions. The submitting will not proceed till trying all the questions. The grading scale of each Instructor has been generated in excel file after submitting and proceeding the application. The request from QECClient.JAVA sends to the server, the server loads that file obtained from the client, the file loads all the system's data i.e., Personal computer name for security purpose on the server and interact with the database. When all the data load on the server successfully, the server generates the response and sends to the client in the shape of Message Dialog Box. Fig. 3 shows the flow of the main screen.

When the student fills the teacher evaluation form completely, the entire teacher's related data loads on the server. Then an excel report is generated on the server which contains the grading scale of every teacher of the specific department which is already being selected by the administrator. The excel report makes accurate and optimized information of the teacher. 


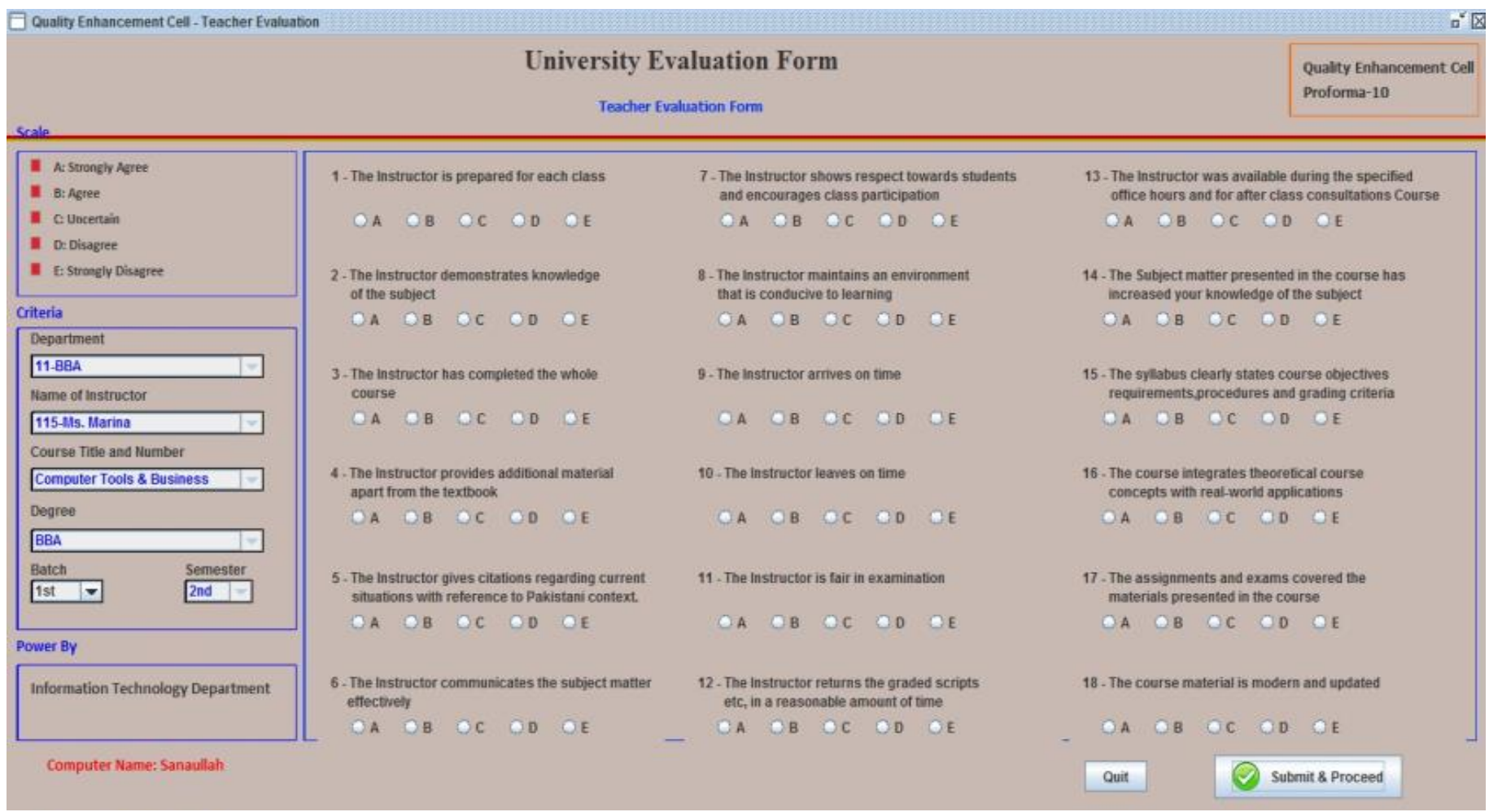

Fig. 2. QEC Main Screen.

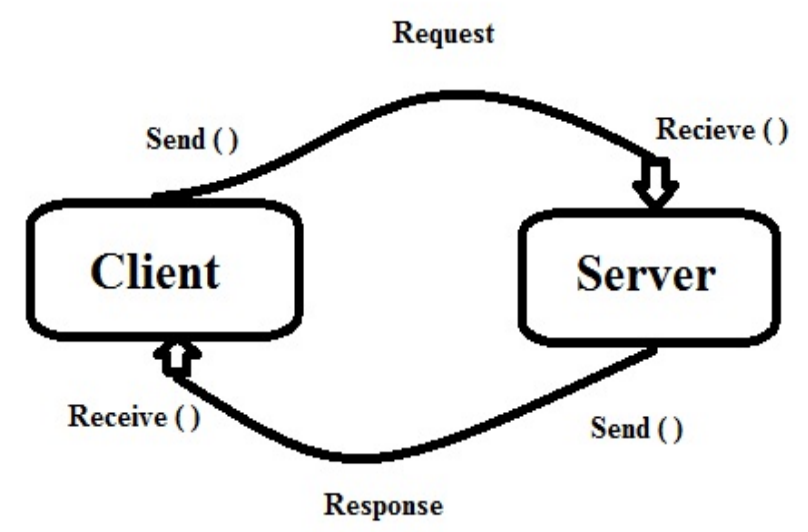

Fig. 3. Flow of the Main Screen.

\section{ADMIN PANEL SCREEN}

Admin panel plays a vital role in updating and managing relevant data or information of the teacher evaluation feedback through web administrator. The administrator uses admin panel screen which contains admin login module. The module provides two textboxes, his/her login id, and password as shown in Fig. 4.

When the administrator successfully login, the control panel screen is visible on the mainscreen which yields the provision to manage QEC application. It produces the facility to enter the facts and statistics collected together for reference of particular batch whose feedback is to be taken. The admin makes changes in department name, batch name, and the name of instructor and course titles. It provides an interface to the client where all these mentioned above parameters are preselected. There is no any chance for the client to makes any changes in the above parameters.

Fig. 5 shows the movement of client's data which shows how data flows from dashboard screen to the server. The data is correctly filled by the client and sends a request to the server. The server stores all the data of the client into the database and generates a response. There is a valid option when filling up the feedback teacher evaluation form. When the data is successfully inserted into the database then it creates a message to the client like this "The Data is Successfully Saved."

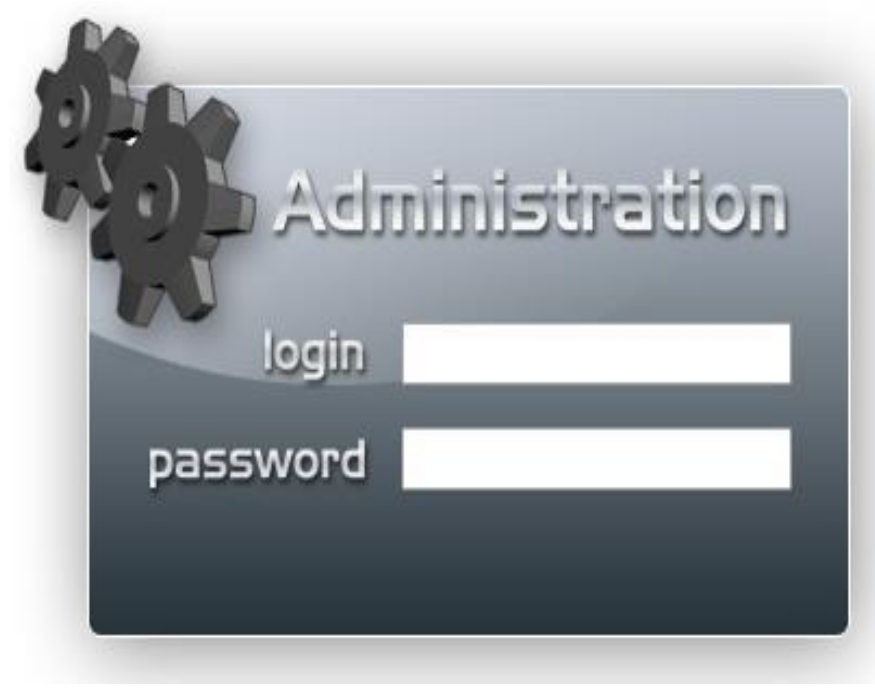

Fig. 4. Circulate of the Panel Display. 


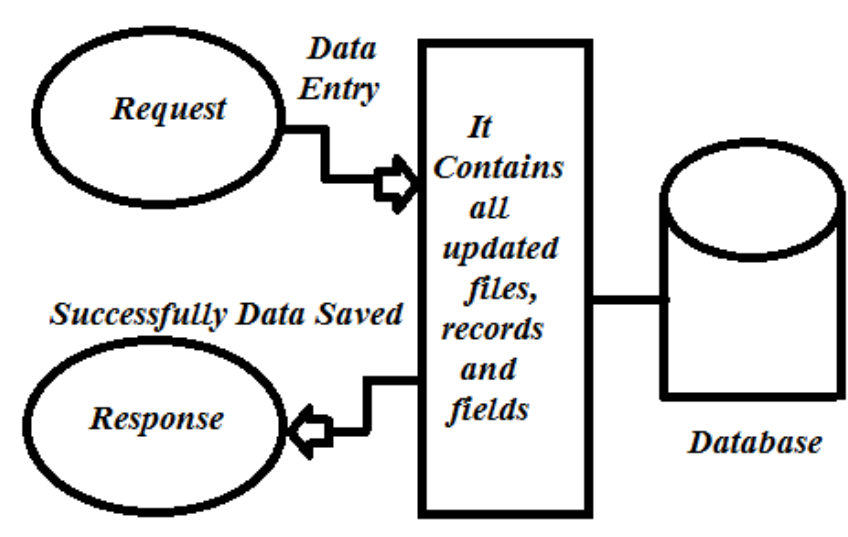

Fig. 5. Flow of Data from Control Panel / Dashboard to Database.

\section{EXPERIMENTS AND RESULTS}

There is need to design and develop QEC applications for taking the proper feedback of teacher evaluation of the departments. The applications are developed in JAVA and PHP computer programming languages. There is too much obligatory to enable the run of applications. Keep application remains in a proper state; the architecture is designed properly of both the applications. There are certain efforts have been needed for obtaining the final results of performance parameters such that design and develop the application. Installation of both applications, JAVA desktop based application, is installed on each machine and web components are placed at the server. To connect each machine with the server, the paper contains same URL Address [IP address / index.PHP]. The paper enables to fetch same size of organization (here same number of students) that may be offline (not connected with internet) locally. To measure the performance parameters of both the applications in terms of average response time, throughput, standard deviation and data transfer rate, there is need of a load balancing testing tool. There are lots of testing tools available for measuring the performance of the desktop application and web-based application. The Apache JMeter testing tool is one among all of them. Jmeter is a popular and powerful free load testing tool which is used for measuring the performance parameters of the applications [16]. The Apache JMeter is the open source testing tool which is used to measure the performance of the server by using the applications. It acts by a surrogate as client side of client/server applications. The server resources like CPU loads, memory loads and response time, is calculated by Jmeter [17]. There is a need to connect load balancing testing tool (Apache Jmeter) with the Server to measure the different performance parameters.

\section{HOW JMETER WORKS}

Jmeter contains a graphical user interface with the clear test plan. To conduct a test, there is a need of test plan in JMeter which describes the steps will take to perform some specific testing task [18]. A test plan is made up of a sequence of test components which determines how the load will be simulated. First, there is a need to build a basic test plan as shown in Fig. 6.

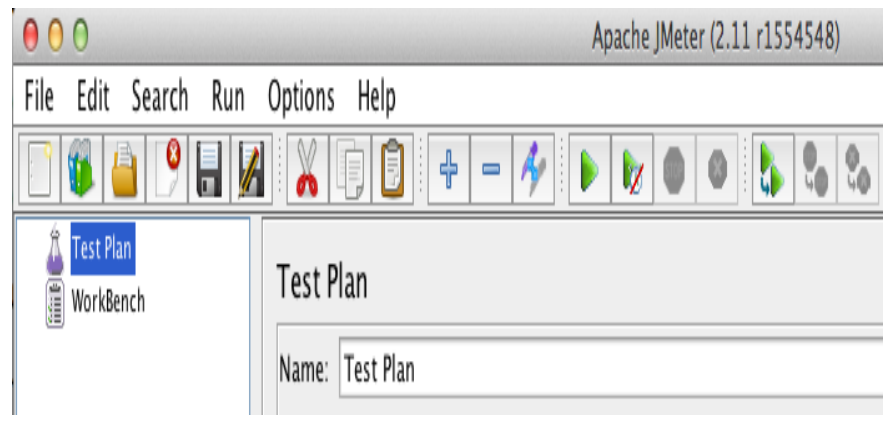

Fig. 6. Build a Basic Test Plan.

The JMeter tool is used to measure the variety of tests which are useful for the environment. A test plan includes the thread group element which is used to identify clearly and definitely the number of running threads and ramp up period [19]. A thread group contains some useful properties that influence the load test such as threads (users), ramp up period and loop count. The loop count shows the number of times, the test will be executed [20]. Here 1 loop count means each test will be repeated 1 time. The threads contain the number of users which you want to simulate, here we set 50 users. Ramp up period is the duration of time will divide the start of thread over, here we set 10 seconds. Loop count shows the number of times, the loop will be executed; here we set 1 count as shown in Fig. 7.

In test plan, The HTTP default config element is used to set the HTTP default request's values. This parameter is more useful to measure multiple HTTP requests on the same server. If the web server runs on the local machine, then there is no need to set the IP address as shown in Fig. 8.

\section{Thread Properties}

Number of Threads (users): 50

Ramp-Up Period (in seconds): 10

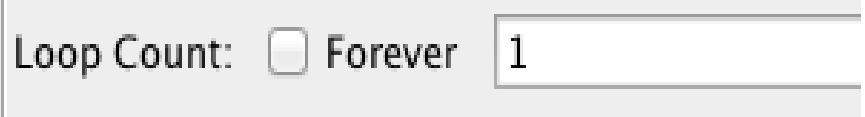

Fig. 7. Thread Properties.

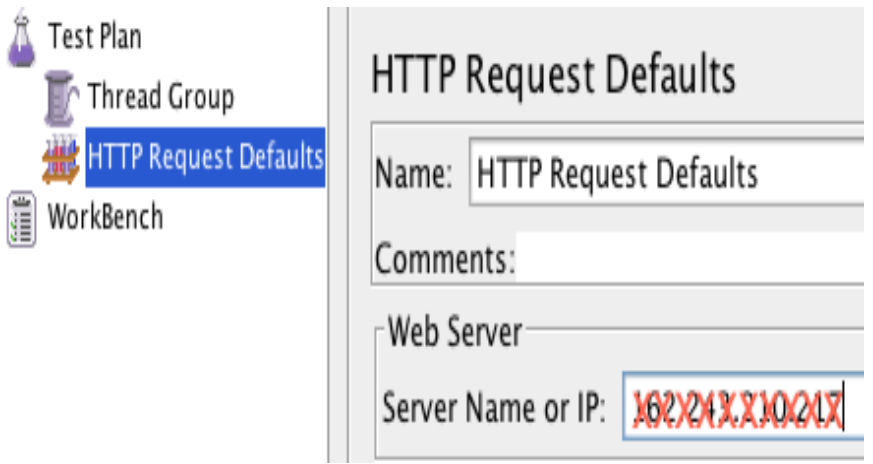

Fig. 8. Http Request Default. 


\section{SUMMARY REPORT IN APACHE JMETER}

The summary report in apache jmeter contains a list of performance parameters which we get after performing some series of steps in apache jmeter. The summary report shows the overall response of the server after sending the requests. The performance parameters are listed below:

1) Label: The label section displays all the recorded HTTP requests.

2) Samples: Samples section shows the number of HTTP requests or the number of users/ threads during the test run.

3) Average response time: Average response time refers to the amount of time; the server takes and returns the response to the user. It is the average response time of HTTP requests and is calculated in milliseconds.

4) Min average response time: It is a minimum amount of response time obtained from the server after sending the HTTP requests. It is also calculated in milliseconds.

5) Max average response time: It is the maximum amount of response time received from the server after sending the HTTP requests. It is too calculated in milliseconds.

6) Standard deviation: It is a quantity which shows how much group members are to be dissimilar from the signify value. A low standard deviation value is also called expected value which expresses the data points are close to the mean value.

7) Error rate: In samples during the run, it shows the error percentage.

8) Throughput: It defines the number of requests per unit of time that the claims which are sent from clients to the server during the test and is calculated either in seconds, minutes or hours.

9) Data transfer rate: It shows the amount of speed through which the data can be emitted from one device to another device and is calculated in megabits or megabytes. Throughput is also another word used for Data Transfer rate.

\section{PERformance Evaluation}

Performance evaluation is a key task to expect when designing and implementing several technologies. Few common complications might occur due to implementation of either lightweight or large IT environments. The load balancing testing tools can be useful computing several parameters of performance of the applications.

\section{A. Performance Evaluation of PHP Application}

The following are the results of QEC application in apache jmeter, which is being designed and developed in PHP programming language. As the application running on the single machine, give the localhost address and port number (8080) when using the apache jmeter. This research paper explains that 300 numbers of samples/users are sent to the server and get the response from the server in the following performance parameters results in apache jmeter.

- $\quad$ Number of Samples/Requests $=300$ requests

- Average Response Time $=33 \mathrm{~ms}=0.033 \mathrm{sec}$
- Min Response Time $=4 \mathrm{~ms}=0.004 \mathrm{sec}$

- Max Response Time $=384 \mathrm{~ms}=0.384 \mathrm{sec}$

- Standard Deviation $=91.71$

- Error rate $=0.00 \%$

- $\quad$ Throughput $=($ number of requests $) /($ total time $)=31.3$ / sec

- Data Transfer Rate $=\mathrm{KB} / \mathrm{sec}=34.81$

\section{B. Performance Evaluation of JAVA Application}

The application which is designed and developed in JAVA programming language shows the response in different parameter results of the server after sending the request of JAVA QEC application in apache jmeter. Socket programming is used inside JAVA because the JAVA application is network based. As the application running on the single machine, an object from the localhost address was sent by using a port number (9090), 300 number of samples/users to the server and get the response back from the server in the following performance parameters results in apache jmeter.

- Number of Samples/Requests $=300$ requests

- Average Response Time $=22 \mathrm{~ms}=0.022 \mathrm{sec}$

- Min Response Time $=4 \mathrm{~ms}=0.004 \mathrm{sec}$

- Max Response Time $=367 \mathrm{~ms}=0.367 \mathrm{sec}$

- Standard Deviation $=61.83$, Error rate $=0.00 \%$

- Throughput $=($ number of requests $) /($ total time $)=$ $31.2 / \mathrm{sec}$

- Data Transfer Rate $=\mathrm{KB} / \mathrm{Sec}=34.64$

\section{XV.COMPARING OF PHP AND JAVA APPLICATION IN TERMS OF PERFORMANCE PARAMETERS}

To compare the graphs, and the best practices help the designers and programmers for selecting the visualization techniques and also allows users to interpret and perform related tasks of how graphs are used to gather. The graph which select must support the user's primary and secondary tasks. If the overall data support these tasks with the same measurement unit than plot both number of events in a single graph. The data or information which changes continuously over time, the Line Graph is a better approach to increase or decrease in data and to display the data or information. A commonly used, line graph contains a series of data points which are connected through the straight line on two axes and changes over time.

\section{- Line Graph in Terms of Average Response Time}

Here the paper compares the server response in line graphs of both PHP and JAVA applications in terms of Average response time. Average response time shows the average amount of time that the server takes, a client must wait before request before getting a response from the server. Different response of server had gotten after sending the requests to the server time by time. By using PHP application, sent 50 number of requests or samples to the server, got average response time in milliseconds which is $48.75 \mathrm{~ms}$. This paper defines when 
sent 300 number of requests to the server, got another result which is $33 \mathrm{~ms}$. In the same way, by using the network-based JAVA application when sent 50 number of requests to the server, got $43 \mathrm{~ms}$ of average response time, but when sent 300 numbers of requests to the server, got different average response time which is $22 \mathrm{~ms}$ as shown in Fig. 9.

\section{Average Response Time}

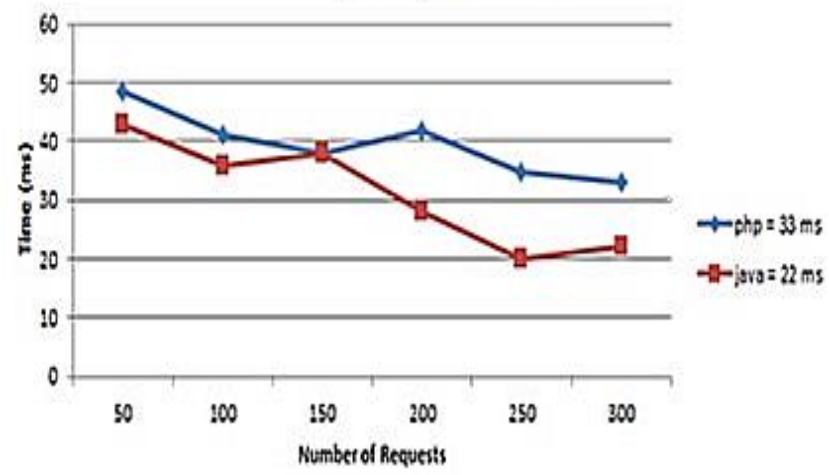

Fig. 9. Line Graph in Terms of Average Response Time.

- Line Graph in Terms of Throughput

Throughput is defined as the measure of units of information; the system can process in a specific period of time or in other words, it can be described as the number of requests per unit time. Here it is calculated in seconds. The different throughput of the server had gotten after sending the requests to the server time by time. By using PHP application, when sent 50 numbers of requests or samples to the server, got throughput in seconds which is 5 seconds. When sent 300 numbers of requests to the server, obtained 31.3 seconds. In the same way, by using the network-based JAVA application when sent 50 numbers of requests to the server, get 5 seconds of throughput, but when sent 300 numbers of requests to the server, got different throughput which is 31.2 seconds. There is little bit variation in performances of both applications, as shown in Fig. 10.

\section{Throughput}

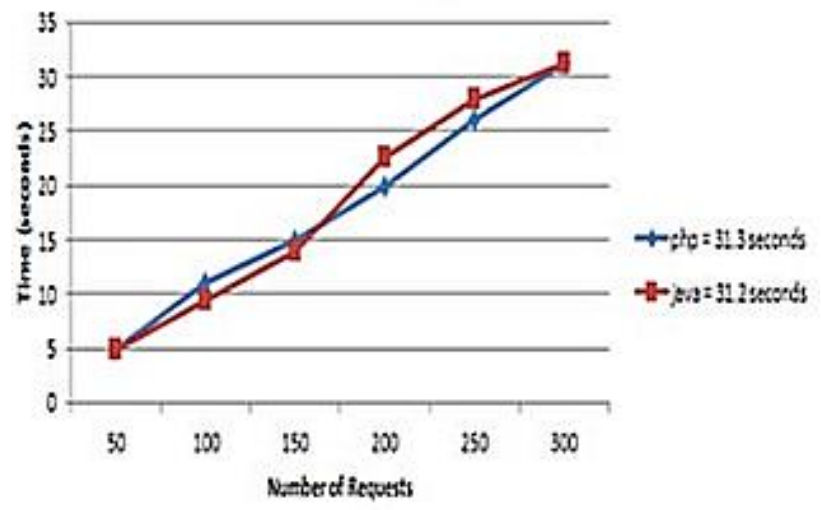

Fig. 10. Line Graph in Terms of Throughput.

\section{- Line Graph in Terms of Standard Deviation}

This paper compares the server response in the line graph of both PHP and JAVA applications in terms of standard deviation. Standard deviation is defined as a measure which is used to liberate the amount of difference of a lay of data values. The data points close to the expected value express the low standard deviation. Different standard deviation values of the server have gotten after sending the number of requests to the server time by time. By using PHP application, when sent 50 numbers of requests or samples to the server, got standard deviation which is 100 data values. When sent 300 numbers of requests to the server, obtained 91.71 data values. In the same way, by using the network-based JAVA application when sent 50 numbers of requests to the server, got 78 data values of standard deviation, but when sent 300 numbers of requests to the server, got different standard deviation which is 61.83 data values, as shown in Fig. 11.

\section{Standard Deviation}

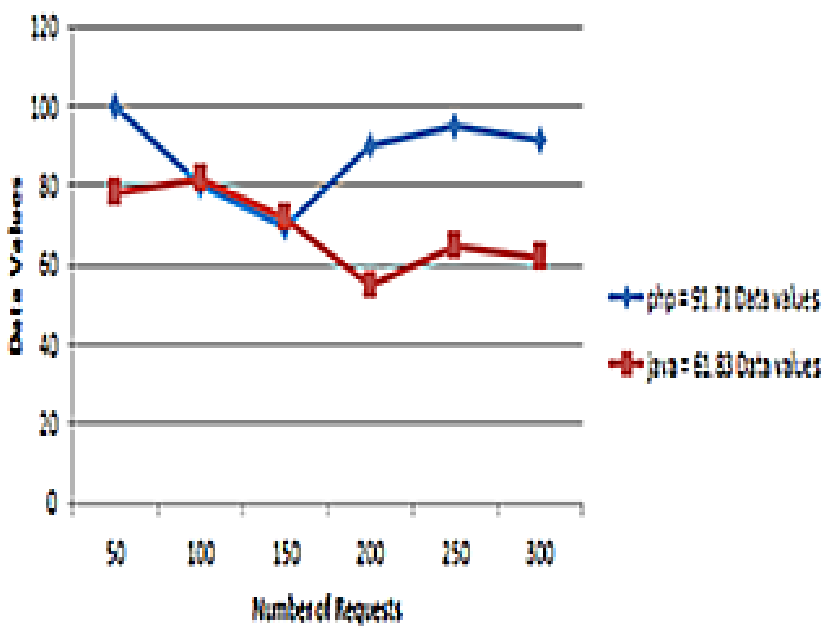

Fig. 11. Line Graph in Terms of Standard Deviation.

\section{- Line Graph in Terms of Data Transfer Rate}

This paper compares the server response in the line graph of both PHP and JAVA applications in terms of data transfer rate. The data transfer rate shows the speed of transmitting the data from one device to another device. It is measured in megabits or megabytes per second. Different data transfer rate values from the server have gotten after sending the number of requests to the server. By using PHP application, when sent 50 numbers of requests or samples to the server, got $40 \mathrm{~kb}$ per second of data transfer rate. When sent 300 numbers of requests to the server, obtained $34.81 \mathrm{~kb}$ per second of data transfer rate. In the same way, by using the network-based JAVA application when sent 50 numbers of requests to the server, get 48 kilobytes per second of data transfer rate, but when sent 300 numbers of requests to the server, got different data transfer rate which is $34.64 \mathrm{~kb}$ per second, as shown in Fig. 12. 


\section{Data Transfer Rate}

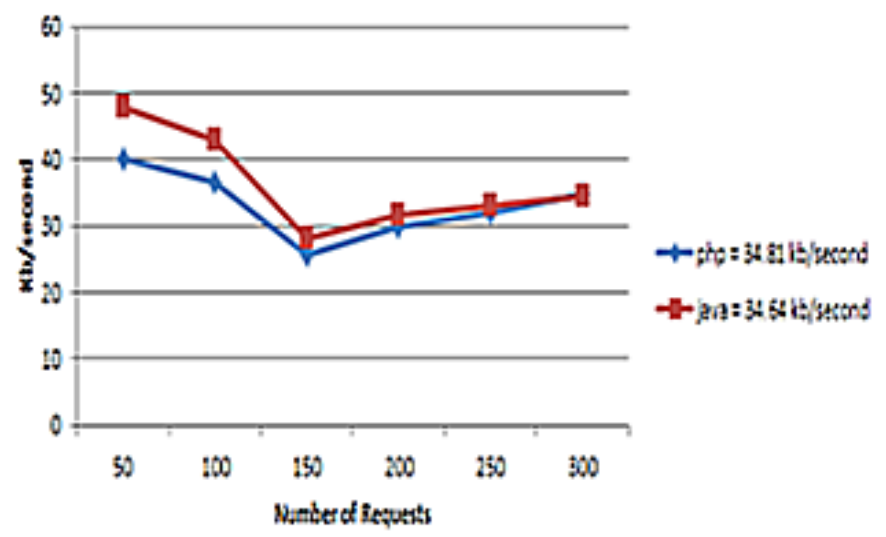

Fig. 12. Line Graph in Terms of Data Transfer Rate.

\section{ACHIEVEMENTS}

All the objectives achieved successfully which are:

- Design and Develop QEC Network Based JAVA Application

- Design and Develop QEC Web Based PHP Application

- Compare the results of both Applications in terms of Average Response Time, Throughput, Standard Deviation and Data Transfer Rate Parameters of the server.

In the results of objectives, obtained the desired aim that is:

- To Find the most feasible solution for getting feedback data for QEC

\section{CONCLUSION}

Now a day, the software technology is growing rapidly day by day. Each and every organization provides facility to the user to run their system correctly takes less time and gives better and accurate results which are beneficent for the organization. All the required information is collected from the clients and has to know which type of application is needed for the organization. The software architects provide proper solution according to the user requirements. By comparing the server results of network-based JAVA application and webbased PHP application in terms of performance parameters, i.e., average response time, throughput, standard deviation and data transfer rate, it is observed that JAVA application is the best feasible solution for feedback evaluation of the organization. With the results of average response time and throughput, we concluded that the JAVA application gives quick response than PHP.

The network-based JAVA application contains fewer data values of the standard deviation as compared to the web-based PHP application. By using the apache jmeter and with the core study of standard deviation, concluded is that the applicationhasfewer data values of standard deviation will give the better influences. So JAVA application is also better than PHP in terms of standard deviation, it contains less standard deviation. The apache jmeter also keeps the feature of data transfer rate and calculate it in kilobytes per seconds. There is a little bit different but almost same in data transfer rate of both the applications. By comparing the results of each and every parameter, it is concluded that network-based JAVA application is better than web-based PHP application in all aspects for the organization.

In future, the educator's testament assessment will make made by utilizing these sorts about electronic framework requisitions. It holds ton of characteristics and gives great deal of profits of association such provides for fast reaction what's more generates exact what's more optimized report card about educator's testament. This electronic framework gives preferred comes about what's more certain ways appears to be will be more positive position over manual framework for QEC.

\section{DISCUSSION}

It may be as a relatable point to each and each association moves towards on the most recent innovations whose use will be ended up additional beneficent for future worth of efforts that is whichever oversaw economy system, majority of the data system, sound alternately feature imparting also a great deal a great amount. Each association needs on move starting with manual framework should programmed framework by utilizing the web built innovations. Our research inspiration is related on the execution from claiming QEC requisitions uncommonly outlined also created over system built JAVA provision and web based PHP provision. The provisions hold the same eighteen inquiries viewing with those educator's testament assessment concerning illustration for every strategy for higher education commission gives the web submission about educator's testament assessment manifestation. In the event for outline judgment report, both requisitions produce exact what's more optimized report card. Anyway here fundamental objective will be should measure the server execution of both requisitions as far as execution parameters like Average response time, throughput, standard deviation and data transfer rate. The load balancing testing tool Apache JMeter is selected for getting the final performance parameters results. The number of requests is sent to the server. When the requests successfully received by the server generate response for the client in different measurement units in milliseconds, seconds and microseconds.

\section{ACKNOWLEDGMENT}

This research has been conducted in Mehran University of Engineering and Technology (MUET) as part of Master's dissertation.

\section{REFERENCES}

[1] Liang, Zhu, et al. "ArcObjects-based eco-environmental data management information system for Three Gorges Project." IEEE International Conference on Information Technology and Computer Science (ITCS 2009), Vol. 2.

[2] Brodie, Michael L., and John Mylopoulos, eds. On knowledge base management systems: integrating artificial intelligence and database technologies. Springer Science \& Business Media, 2012.

[3] Ghanem, Thanaa M., and Walid G. Aref. Databases deepen the web. IEEE Computer 37.1 (2004): 116-117.

[4] Van Dijk, Jan AGM, Oscar Peters, and Wolfgang Ebbers. "Explaining the acceptance and use of government Internet services: A multivariate 
analysis of 2006 survey data in the Netherlands." Government Information Quarterly 25.3 (2008): 379-399.

[5] Walden, James, et al. "Idea: JAVA vs. PHP: security implications of language choice for web applications." International Symposium on Engineering Secure Software andSystems.Springer Berlin Heidelberg, 2010.

[6] Wright, William, and Dana Moore. "Agile language development: the next generation." IEEE Aerospace Conference, 2006.

[7] Moberg, Åsa, et al. "Printed and tablet e-paper newspaper from an environmental perspective, A screening life cycle assessment."Environmental Impact Assessment Review 30.3 (2010): 177191.

[8] Hoshino, Yuta, et al. "An on-line algorithm to determine the location of the server in a server migration service." 2015 12th Annual IEEE Consumer Communications and Networking Conference (CCNC). IEEE, 2015.

[9] Mietzner, Ralph, Frank Leymann, and Mike P. Papazoglou. "Defining composite configurableSaaS application packages using SCA, variability descriptors and multi-tenancy patterns. Third IEEE International Conference on "Internet and Web Applications and Services, 2008 (ICIW'08).

[10] Ross, Adam M., Donna H. Rhodes, and Daniel E. Hastings. "Defining changeability:Reconciling flexibility, adaptability, scalability, modifiability, and robustness for maintaining system lifecycle value." Systems Engineering 11.3 (2008): 246-262.
[11] Cui, Wei, et al. "The research of PHP development a framework based on MVC pattern. Fourth IEEE International Conference on Computer Sciences and Convergence Information Technology, ICCIT'09.

[12] Loo, Alfred Wai-Sing. "JAVA Network Programming." Peer-to-Peer Computing. Springer London, 2007,67-90.

[13] Harold, Elliotte Rusty. JAVA network programming. "O'Reilly Media, Inc.", 2013.

[14] Curino, Carlo A., et al. "Schema evolution in Wikipedia: toward a web information system benchmark." In International Conference on Enterprise Information Systems (ICEIS) 2008.

[15] Pernencar, Cláudia, Teresa Romão, and Graça Rocha Simões. "The design process of an e-Health project: Applying the HSI framework for interface analysis." Serious Games and Applications for Health (SeGAH), 2016 IEEE International Conference on. IEEE, 2016.

[16] JMeter, Apache. "Apache software foundation." (2010).

[17] Team, JMeter. Enhancement of JMeter. Diss. Indian Institute of Technology, Bombay Mumbai, 2013.

[18] Kurniawan, Budi, and JMeteris a JAVA. "Using JMeter." http:/onJAVA.com/Ipt/a/3066,(Jan. 15, 2003) (2003):1-9.

[19] Nevedrov, Dmitri. "Using JMeter to Performance Test Web Services. "Published on 57 dev2dev (http://dev2dev. bea. com/) (2006).

[20] Alsmadi, Izzat, and SaschaAlda. "Simulation Based Load Testing In Web Services." The 6th International Conference on Information Technology May, 2013. Vol. 8. 